\title{
Performance Analysis of UAV Cellular Communications
}

\author{
Anandpushparaj $\mathrm{J}^{1}$, Vineeth Palliyembil ${ }^{1}$, Laxman Gattu Nadiminti ${ }^{1}$, Maurizio Magarini ${ }^{2}$, P.Muthuchidambaranathan ${ }^{1}$ \\ ${ }^{1}$ Department of Electronics and Communication \\ National Institute of Technology Tiruchirappalli, Tamilnadu, 620015, India \\ anandece1989@gmail.com, vineethpalliyembil@gmail.com, gnlaxman@gmail.com, muthuc@nitt.edu \\ ${ }^{2}$ Dipartimento di Elettronica, Informazione e Bioingegneria, Politecnico di Milano, Milan, Italy \\ maurizio.magarini@polimi.it
}

\begin{abstract}
The days that are to come where remote-controlled aerial vehicles can occupy our skies are quick approaching thanks to the introduction of efficient and reliable tiny aerial vehicles and therefore the increasing demand to be used of such vehicles in an exceedingly excess of civil applications. It is necessary to grasp the characteristics of networks with aerial vehicles to deploy multiple UAVs into the air traffic in a secured and safe manner. In this paper, we analyze the downlink channel performance of cellular connected UAVs located at different heights. We determine the average path loss that can be occurred from the base station to an aerial vehicle located at different heights. We observe that due to the line of sight links interference power is more which leads to poor signal to interference plus noise ratio. In order to increase the throughput and suppress the interference, we employ linear precoding at the transmitter side by assuming perfect channel state information at the base station. We determine the data rates that can be achieved over a $10 \mathrm{MHz}$ system bandwidth in downlink when various precoding techniques are applied at transmitter base station. Our results conclude that the achievable data rates decline as the UAV reaches higher altitudes due to more interference power receiving from other base stations.
\end{abstract}

Keywords-Unmanned Aerial Vehicle(UAV), Base station(BS), line of sight(LOS), downlink, A2G, path loss, interference, Precoding, 3GPP

\section{INTRODUCTION}

A far-reaching utilization of pilotless unmanned aerial vehicles (UAVs) like drones, tiny aircrafts, and airships in civil applications is directly being empowered by ongoing advances in the design of reliable and cost-effective drone technology. Applications include military, agriculture, cargo delivery, surveillance and monitoring, telecommunications, search and rescue operations and many others [1]. UAVs can be used as aerial base stations [2] to provide reliable, cost-effective and emergency communication to the desired regions when they are properly deployed. In order to acquire the above technological visions into reality, there is a need to establish reliable communication between UAVs and ground base stations (BS). In this scenario UAV acts as new aerial user equipment (AUE) connected to the cellular network in addition to the ground users (GU) and hence the name 'UAV cellular communications'.

UAV cellular communications serve mainly two purposes (1) Command \& Control to operate these UAVs safely and autonomously (2) Data communication in which high-speed information rates for sanctioning real-time delivery of measuring information or high-resolution photographs. There have been numerous research studies conducted for the wireless connectivity of AUEs with an existing cellular network. It seems that interference turns out to be the major limiting issue for the UAV cellular communication due to the fact that current cellular systems are designed for ground users (GUE) only. A2G channel propagation conditions are completely different from that of terrestrial due to the LoS communication between AUEs and BSs. For downlink channel i.e. from BS to UAV, UAV receives more interference with high probability from other base stations which are not associated with it as UAV reaches high altitude [3]. Hence the downlink performance of AUE is poorer compared to terrestrial users. Thus, formulating effective interference mitigation techniques by considering unique $\mathrm{BS}-$ UAV channel is crucial for UAV cellular Communications.

Third Generation Partnership Project (3GPP), accountable for normalizing global cellular network standards has concluded a study object [4] "Enhanced Support for Aerial Vehicles" aimed towards making ready LTE networks to support UAV cellular communications for the UAVs flying at heights up to $300 \mathrm{~m}$ above the ground. 3GPP works to incorporate the development of LoS probability models and propagation channels including coverage and capacity assessments that can be provided by cellular connected UAVs. In this paper, for various heights of aerial vehicle path loss measurements are carried out and also determine the achievable channel rates for the UAV downlink C\&C channel with various precoding schemes.

\section{AIR TO GROUND CHANNEL MODELING}

UAV cellular communication is naturally affected by the medium between BS and UAV. Accurate modeling of Air to Ground channel is needed to establish reliable communication between them. High UAV altitudes result in distinctive channel characteristics due to robust LoS links. Compared to terrestrial systems Path loss from a base station to UAV depends on the location of UAV, the height of UAV and type of propagation environment (rural, urban, semi-urban etc.). A2G communication links are often either LoS or NLoS. In this paper, we consider the height dependent 3GPP probabilistic model in which LoS and NLoS links are considered separately with a different probability of occurrences. 


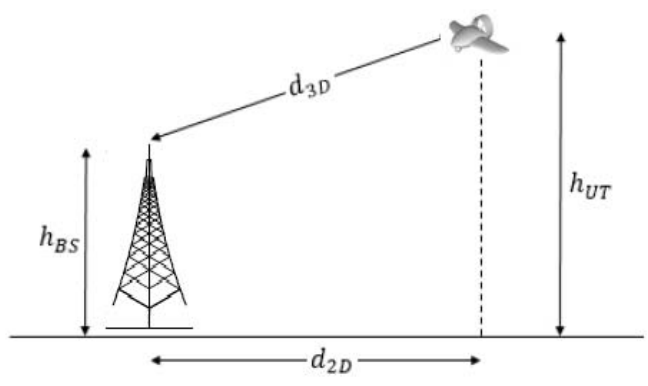

Fig. 1. Understanding 2D and 3D distances of UAV

The 3D distance between UAV and the BS can be defined as below

$$
d_{3 D}=\sqrt{\left(d_{2 D}\right)^{2}+\left(h_{B S}-h_{U T}\right)^{2}}
$$

where $h_{B S}$ and $h_{U T}$ represent the height of the base station and height of UAV respectively.

The average path loss can be considered as

$P L_{\text {avg }}=\mathrm{P}(\mathrm{LoS}) \times P L_{L O S}+(1-\mathrm{P}(\mathrm{LoS})) \times P L_{N L O S}$

Where $\mathrm{P}(\mathrm{LoS})$ is the probability of having a line of sight between the aerial user and the base station. $\mathrm{PL}_{\mathrm{LOS}}$ and $\mathrm{PL}_{\mathrm{NLOS}}$ denote path loss due to LoS and NLoS respectively which depends on transmitter and receiver heights.

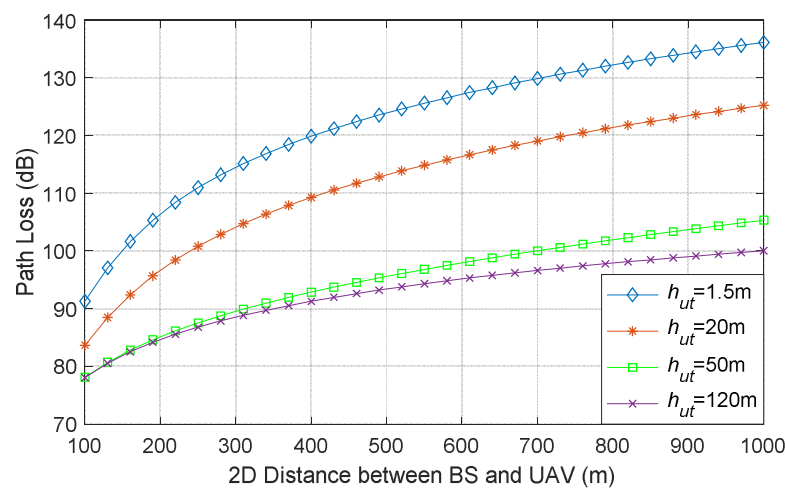

Fig. 2. Plot showing Path Loss vs 2D distance between BS and UAV for different heights of aerial vehicle

From the above result (Fig2), it is clear that when the height of the UAV increases path loss gets reduced up to a certain height of $120 \mathrm{~m}$. Reduction of path loss with aerial height has been verified in a live LTE network in [5]. From 3GPP study [4], shadow fading variation gets also reduced with the height of UAV. Both of these observations conclude that the propagation channel between BS and UAV is clear from obstacles as UAV reaches high altitudes which lead to increase in the received signal strength at the UAV and also results in stronger interference in both uplink and downlink. We observe that propagation channel becomes independent of height beyond $120 \mathrm{~m}$ since though height is increased, the radio path clearance will not improve further.

\section{SYSTEM MODEL}

Consider a Massive MIMO cellular network in which BSs are deployed on the hexagonal layout with B base stations equipped with a large number of antennas providing coverage to its associated users. Unlike terrestrial cellular network, the network under beneath serves both ground users as well as aerial users.

Each BS consists of $N_{b}$ antennas with transmission power $P_{b}$ which serve certain $\mathrm{U}$ number of uniformly distributed ground $\left(N_{b} \gg U\right)$ users as well as single UAV with one receiving antenna (BS serves single aerial user including ground users). In our simulations, we consider UAV downlink communication in which height of the UAV $\left(h_{U T}\right)$ is uniformly distributed between $1.5 \mathrm{~m}$ and $300 \mathrm{~m}$ over $2 \mathrm{GHz}$ carrier frequency ( $\mathrm{fc}=2 \mathrm{GHz}$ ) with the height of the base station as $25 \mathrm{~m}$. we consider the 3GPP urban macro (UMa-AV) scenario with inter-site distance (ISD) as $500 \mathrm{~m}$. We assume all UAVs are located at a certain 2D distance of $100 \mathrm{~m}$ from their respective base stations.

\section{A. Channel Model}

Channel can be modeled as Rayleigh for terrestrial communications in case of urban areas. However, A2G communications have totally different characteristics. Due to the presence of LoS links, small scale fading of the A2G channel experiences Rician fading which consists of a rician factor $\mathrm{k}$. In the case of suburban areas, LoS signals are weaker than reflected signals so Rayleigh fading is considered [6]. In this paper, we tend to adopt a Rician channel model with rician $\mathrm{k}$ factor dependent on the height of UAV [4] which has both LoS and NLoS paths. Hence, the channel gain matrix can be depicted as [6]

$$
H=\sqrt{\frac{k}{k+1}} H_{L o S}+\sqrt{\frac{1}{k+1}} H_{N L O S}
$$

Where $H_{L o S}$ and $H_{N L O S}$ represent channel matrix for LoS and NLoS communication.

Let us consider the channel vector between base station $\mathrm{b}$ and user $\mathrm{u}$ in $k^{t h}$ cell as $h_{b k u} \in C^{N_{b} x 1}$. We denote the BS b serves a set of users $U_{b}$.

Estimated channel matrix can be represented as $H_{b} \in C^{N_{b} x U_{b}}$

$$
H_{b}=\left[h_{b b_{1}}, h_{b b_{2}} \ldots \ldots . h_{b b_{U_{b}}}\right]
$$

Precoder matrix $W \in C^{N_{b} x U_{b}}$ is given by

$$
W_{b}=\left[w_{b b_{1}}, w_{b b_{2}} \ldots \ldots . . w_{b b_{U_{b}}}\right]
$$

The received signal $y_{b u}$ to the user $u$ of base station $b$ can be expressed as

$$
\begin{aligned}
y_{b u}= & \sqrt{P_{b}} h_{b b u}^{H} w_{b u} s_{b u}+\sqrt{P_{b}} \sum_{i \epsilon U_{b} / u} h_{b b u}^{H} w_{b i} s_{b i}+ \\
& \sqrt{P_{b}} \sum_{j \in B / b} \sum_{i \epsilon U_{j}} h_{j b u}^{H} w_{j i} s_{j i}+\epsilon_{b u}
\end{aligned}
$$

$h_{b b}$ : Channel coefficient between serving BS and UAV in cell b

$h_{j b}$ : Channel coefficient between other interfering BSs from other cells in cell $b$

$s_{b u}:$ Intended signal for user $\mathrm{u}$ in cell $\mathrm{b}$

$w_{b u}: C^{N_{b} x 1}$ transmit precoding vector utilized by BS $\mathrm{b}$ in order to serve user $\mathrm{u}$

$\epsilon_{b}:$ Thermal noise

The right-hand side of (6) consists of four terms. First-term represents the intended signal, second-term represents intra 
cell interference caused due to the other users of serving BS, third -term represents inter-cell interference from other BSs and the last term represents thermal noise.

The resulting instantaneous signal-to-interference-plusnoise ratio (SINR) $\gamma_{b u}$ experienced by user $\mathrm{u}$ associated to $\mathrm{BS} \mathrm{b}$ is given by

$$
\gamma_{b u}=\frac{P_{b}\left|h_{b b u}{ }^{H} w_{b u}\right|^{2}}{P b \sum_{i \in U b / u}\left|h_{b b u}{ }^{H} w_{b i}\right|^{2}+P b \sum_{j \in B / b} \sum_{i \in U j}\left|h_{j b u}{ }^{H} w_{j i}\right|^{2}+\sigma_{\epsilon}{ }^{2}}
$$

TABLE 1 . SYSTEM PARAMETERS

\begin{tabular}{|l|l|}
\hline BS inter-site distance & $500 \mathrm{~m}$ \\
\hline BS antenna height & $25 \mathrm{~m}$ \\
\hline BS transmitting antennas $N_{b}$ & 128 \\
\hline Carrier Frequency & $2 \mathrm{GHz}$ \\
\hline System Bandwidth & $10 \mathrm{MHz}$ \\
\hline BS transmission power & $46 \mathrm{dBm}$ \\
\hline Thermal noise & $\begin{array}{l}-174 \quad \mathrm{dBm} / \mathrm{Hz} \quad \text { spectral } \\
\text { density }\end{array}$ \\
\hline $\begin{array}{l}\text { LoS probability, Path loss, } \\
\text { Shadow fading variations }\end{array}$ & As per UMa-AV in [4] \\
\hline
\end{tabular}

\section{PERFORMANCE IMPROVEMENT THROUGH LINEAR DIGITAL} PRECODING

Among various aspects of performance and design issues of massive MIMO systems, precoding at the BS is one in all the foremost necessary factors so as to confirm reliable downlink transmission. Precoding techniques are classified into linear and nonlinear. As massive MIMO employs the large number of antennas nonlinear precoding schemes suffer from high complexness that makes them impractical in spite of their optimal performance [8]. In this paper, we analyze the downlink channel performance of UAV when linear precoding techniques [7] such as Zero-forcing (ZF) precoding, Matched Filter (MF) precoding and Regularized Zero Forcing precoding (RZF) are employed at the base station. Achievable throughput at various heights of UAV is determined when these precoding techniques are enabled.

The performance of precoding is mainly affected by channel state information at the transmitter BS. We assume that transmitter BS has perfect channel state information and precoding techniques are implemented to evaluate the performance.

\section{B. MF precoding}

Matched Filter (MF) precoder can be simply termed as the downlink channel matrix which is given by

$$
W_{M F}=\sqrt{\beta} H
$$

To normalize the signal power we use scaling factor $\beta$ which can be treated as

$\beta=\frac{1}{\operatorname{tr}\left(H^{H} H\right)}$

The received signal vector can be represented as

$$
y_{M F}=\sqrt{P_{b} \beta} H^{H} H s+n
$$

MF precoder can also be termed as Maximum Ratio Transmission (MRT) precoder that maximizes the signal power at the meant user.

\section{ZF Precoding}

$\mathrm{ZF}$ precoding is another precoding technique which wipes out the interference by transmitting the signal to the meant user while nulling within the directions of other users.

$$
W_{Z F}=\sqrt{\beta} H\left(H^{H} H\right)^{-1}
$$

The received signal vector can be represented as

$$
y_{Z F}=\sqrt{P_{b} \beta} H^{H} H\left(H^{H} H\right)^{-1} s+n
$$

\section{Regularized ZF Precoding}

Precoding vector of RZF precoding can be represented as

$$
W_{Z F}=\sqrt{\beta} H\left(H^{H} H+\chi I_{U_{b}}\right)^{-1}
$$

The received signal vector can be represented as

$$
\begin{aligned}
& y_{Z F}=\sqrt{P_{b} \beta} H^{H} H\left(H^{H} H+\chi I_{U_{b}}\right)^{-1} s+n \\
& \text { V. NUMERICAL RESULTS AND DISCUSSIONS }
\end{aligned}
$$

We consider $N_{b}=128$ antennas at the base station and UAV has one receiving antenna. Shadow fading variations which vary for LoS and NLoS [4] are considered. We assume UAV associated to its serving BS receives interference from 6 other base stations where each BS serves around 16 users in which one user is aerial user and other 15 are ground users. We include path loss and shadowing fading models [4] in calculating the intra cell interference and inter-cell interference. We determine the channel capacity over a system bandwidth of $10 \mathrm{MHz}$ for various precoding schemes.

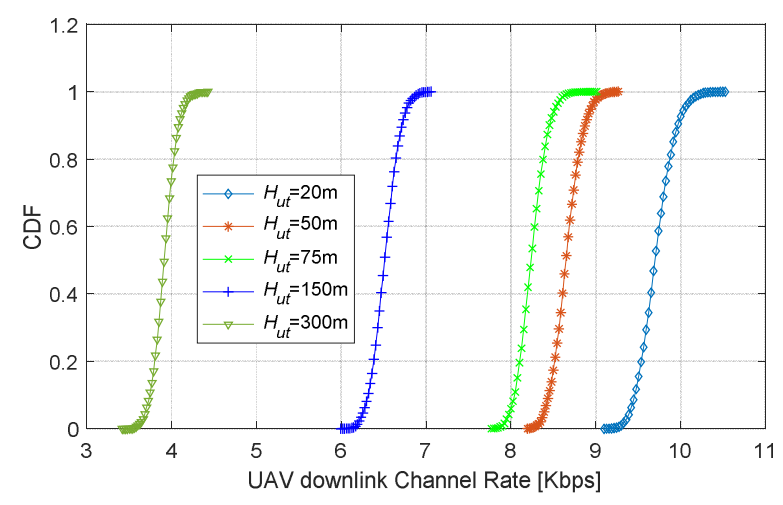

Fig. 4. UAV downlink channel rate vs CDF when MF precoding is applied as a function of UAV height

Fig. 4 shows the cumulative distribution of downlink channel rates that can be obtained for UAV heights of $20 \mathrm{~m}, 50 \mathrm{~m}, 75 \mathrm{~m}$, $150 \mathrm{~m}$, and $300 \mathrm{~m}$ when MF precoding is implemented. MF precoding can increase the received signal power but it does not suppress the interference and hence signal to interference plus noise ratio is still remains poor and hence achievable data rates would be in the order of kbps. From fig. 4 we observe that channel capacity gets reduced with the increase in the height of the UAV. This is mainly due to the increase in the interference power from intra cell users and other base stations when UAV reaches higher altitudes. 


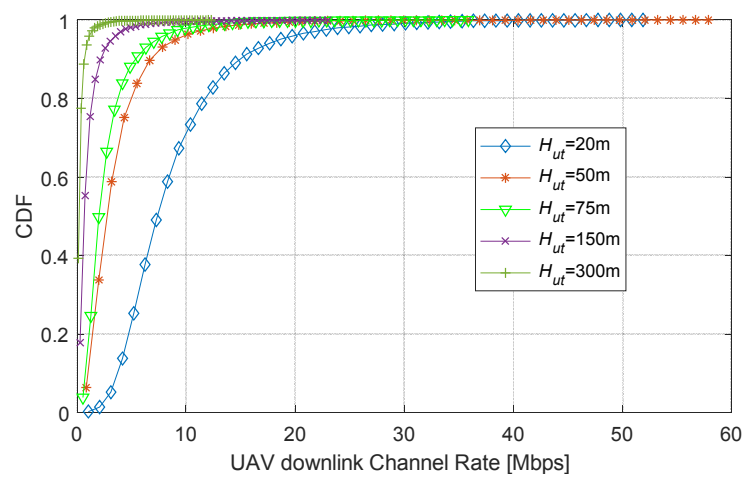

Fig. 5. UAV downlink channel rate vs CDF when ZF precoding is applied

Fig. 5 shows that we can achieve downlink channel rates in the order of Mbps when ZF precoding is applied. This is due to the suppression of intra cell interference caused by the ground users of same serving BS which leads to improved signal to interference noise ratio. Achievable data rates are low when UAV reaches altitudes beyond $100 \mathrm{~m}$.

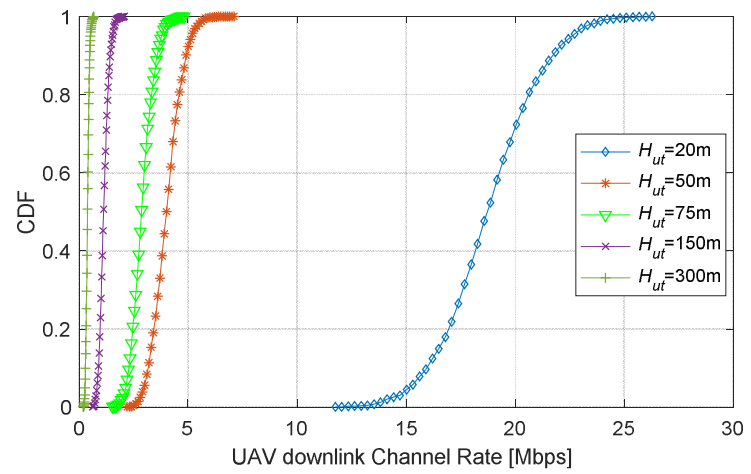

Fig. 6.UAV downlink channel rate vs CDF when Regularized ZF precoding is applied as a function of UAV height

Regularized ZF precoding gives slightly better channel rates compared to $\mathrm{ZF}$ precoding. Fig. 6 shows channel capacity declines with the increase in height. CDF curve tells that RZF precoding gives reasonable data rates in most of the cases than that of ZF precoding.

\section{CONCLUSION}

Performance of UAV cellular communication when BSs are employed with a large number of antennas has been analyzed by considering standard 3GPP path loss models. The most important aspect to serve aerial users is to control their vulnerability to interference. We examined that path loss gets decreased with UAV height for smaller altitudes. Our analysis tells that by employing transmitter precoding techniques effectively we can eradicate the interference and achieve the reasonable data rates which get reduced with UAV height. ZF and RZF precoding techniques give better channel rates compared to MF precoding.

\section{ACKNOWLEDGMENT}

This paper was supported under the Indo-Italy researcher exchange program for 2017-2020 (INT/ITALY/P-6/2016 (ER)).

\section{REFERENCES}

[1] Y. Zeng, R. Zhang, and T. J. Lim, "Wireless communications with unmanned aerial vehicles: opportunities and challenges," IEEE Communications Magazine, vol. 54, no. 5, pp. 36-42, 2016.

[2] M. Mozaffari, W. Saad, M. Bennis, Y.-H. Nam, and M. Debbah, "A tutorial on UAVs for wireless networks: Applications, challenges, and open problems," available online: arxiv.org/abs/1803.00680, 2018

[3] K. Welch, "Evolving cellular technologies for safer drone operation," Qualcomm 5G White Paper Present., San Diego, CA, USA, Tech. Rep., Oct. 2016.

[4] 3GPP Technical Report 36.777, "Technical specification group radio access network; Study on enhanced LTE support for aerial vehicles (Release 15)," Dec. 2017

[5] R. Amorim et al., "Radio channel modelling for UAV communication over cellular networks," IEEE Wireless Commun. Lett., vol. 6, no. 4, pp. 514-517, Aug. 2017.

[6] A. Paulraj, R. Nabar, and D. Gore, Introduction to Space-Time Wireless Communications. Cambridge, U.K.: Cambridge Univ. Press, 2003.

[7] Fatema N., Hua G., Xiang Y., Peng D., Natgunanathan I. Massive MIMO Linear Precoding: A Survey. IEEE Syst. J. 2018;122:3920-3931.

[8] Varna L. Babu, Luxy Mathews, Sakuntala S. Pillai, "Performance analysis of linear and nonlinear precoding in MIMO systems," Int. J. Advanced Res. Computer Commun. Eng. 4, No. 6, 373 (2015)

[9] W. Khawaja, I. Guvenc, D. W. Matolak, U.-C. Fiebig, and N Schneckenberger, "A survey of air-to-ground propagation channel modeling for unmanned aerial vehicles," available as arXiv:1801.01656, Jan. 2018.

[10] X. Lin, V. Yajnanarayana, S. D. Muruganathan, S. Gao, H. Asplund, H. Maattanen, M. Bergstrom, S. Euler, and Y. . E. Wang, "The sky is not the limit: LTE for unmanned aerial vehicles," IEEE Communications Magazine, vol. 56, no. 4, pp. 204-210, APRIL 2018. 